УДК 622.834

\title{
АНАЛИЗ ПРОЦЕССА ДЕФОРМИРОВАНИЯ ГРУНТОВОЙ ДАМБЫ ПРИ ЗАПОЛНЕНИИ ЧАШИ ХВОСТОХРАНИЛИЩА
}

\author{
Ефремов Евгений Юрьевич1, \\ Efremov-eu@mail.ru \\ Институт горного дела УрО РАН, \\ Россия, 620219, г. Екатеринбург, ул. Мамина-Сибиряка, 58.
}

\begin{abstract}
Актуальность. Мониторине деформаций грунтовых дамб хвостохранилищ - это важный элемент в обеспечении промышленной безопасности. Результатом деформационного мониторинга являются зафиксированные смещения поверхности дамб или отдельных марок деформационной сети. Однако не все фиксируемые смещения являются опасными. В данной работе обосновано разделение фиксируемых смещений на деформации склонов, представляющее опасность прорывов, и оседания вследствие сжатия нижерасположенного массива горных пород, не представляющие опасности.

Целью работы является определение характера механического воздействия заполняемого резервуара хвостохранилища на ограждающую дамбу и окружающую земную поверхность на примере одного из хвостохранилищ. Примененный подход в оценке деформирования грунтовых дамб базируется на соотношениях между наблюдаемыми смещениями, характеристиками объекта и интенсивностью складирования отходов. При этом принципиально важно, что нет зависимости между предлагаемой обработкой измерений и методами определения смещений.

Методы. Измерение и обработка результатов осуществлялись при помощи геодезических методов - геометрического нивелирования и ГНСС позиционирования. Анализ результатов производился с помощью статистических и геостатистических методов.

Результаты и выводы. Установлено, что в течение восьми серий измерений преобладающим типом смещений являются оседания. Анализ измерений показал, что горизонтальные смещения пунктов наблюдательной станции находятся на уровне погрешности измерений. Распределение оседаний марок дамбы хвостохранилища, характер деформирования головной плотины, а также отсутствие значимых горизонтальных смещений позволяют заключить, что тело дамбы находится 8 устойчивом состоянии, а фиксируемые оседания - проявление деформации естественного массива в основании гидросооружения под нагрузкой веса складируемых хвостов.
\end{abstract}

\section{Ключевые слова:}

Грунтовые дамбы, хвостохранилище, прорыв дамб, оседания, устойчивость склонов, наблюдательная станция.

\section{Введение}

Мониторинг десрормаций дамб хвостохранилищ

Аварийность хвостохранилищ представляет серьезную угрозу для жизни людей и инфраструктуры $[1,2]$. Наибольшую известность получила авария, произошедшая 29 января 2019 г. на руднике Córregodo Feijão в городе Брумадинью, Бразилия [3]. В результате прорыва дамбы погибло 270 человек, был уничтожен железнодорожный мост. Экономический ущерб составил 19 млрд долларов.

На данный момент существует достаточное количество работ, посвященных исследованиям возможных причин аварийности хвостохранилищ и грунтовых дамб [4-9] и их последствий [10-12].

Основой безопасной эксплуатации грунтовых дамб и хвостохранилищ является мониторинг состояния гидротехнических сооружений. Мониторинг необходим для того, чтобы вовремя определить нарушение наблюдаемого условия. Помимо прочего, в мониторинг гидротехнических сооружений входят визуальный контроль состояния склонов, контроль уровня грунтовых вод и мониторинг деформаций ограждающих дамб. Среди методов мониторинга деформаций распространены: ГНСС, нивелирование, тахеометры [13-15]. В последние несколько десятилетий становится доступнее мониторинг с помощью спутниковых InSAR технологий [16-18]. В подобных исследованиях, как правило, уделяется много внима- ния деталям обработки результатов и количественным характеристикам и недостаточно внимания интерпретации результатов мониторинга.

Длительный мониторинг деформаций, позволяет отследить медленно продолжающиеся процессы, когда изменения, фиксируемые одной серией наблюдений, находятся на уровне погрешности измерений, однако накапливаются со временем. Для лучшего понимания процессов необходимо анализировать изменения не в отрыве, а совместно с другими данными об объекте. Сами по себе данные мониторинга - это лишь сырые материалы, которые должны быть правильно интерпретированы для адекватного понимания процессов, происходящих в теле грунтовых дамб

Задачи интерпретации смещений

Цель проведенных работ - определение характера механического воздействия заполняемого резервуара хвостохранилища на ограждающую дамбу и окружающую земную поверхность. Определение взаимосвязи между деформацией ограждающей дамбы и мощностью хвостохранилища.

Наиболее опасна потеря устойчивости склонов грунтовых дамб, в то время как смещения, вызванные оседаниями поверхности, не несут прямой угрозы прорыва дамб. Основной вопрос, стоящий перед настоящими исследованиями, - фиксируемые измерения оседания и деформации дамбы определяются свойствами грунтов, из которых состоит ограждаю- 
щая дамба, или свойствами осадочных пород естественного основания хвостохранилища? Иными словами, требуется определить, являются ли наблюдаемые оседания дамбы в большей степени следствием деформирования тела хвостохранилища или следствием оседания земной поверхности и сжатия естественного массива под весом гидросооружения.

Задачи работы заключаются в следующем:

1) определение распределения смещений ограждающей дамбы и головной плотины;

2) определение распределения мощности плотины. Исходные данные - съемка местности, данные урезов воды на хвостохранилище;

3) установление наличия/отсутствия взаимосвязи между распределением мощности хвостохранилища и фиксируемой величиной смещения;

4) установление наличия/отсутствия взаимосвязи между приростом массы хвостохранилища и оседаниями.

\section{Характеристика сооружения}

Общие сведения

Площадь гидроузла составляет около 19 млн кв. м. Склон ограждающей дамбы на данный момент времени занимает 27 \% площади хвостохранилища, по мере увеличения высоты сооружения будет занимать все большую долю обшей площади. Основу хвостохранилища составляет головная плотина, возведенная вкрест овражной балки в 70-х гг. прошлого века, ее длина несколько превышает 2 тыс. м, высота составляет около 130 м. По мере увеличения хвостохранилища возводится ограждающая дамба, предназначенная для создания ёмкости хвостохранилища, ее высота над рельефом колеблется от 5 до 140 м, длина по периметру около 10 тыс. м. Ограждающая дамба и головная плотина возводятся поярусно (высота яруса наращивания 5 м) шагающим экскаватором из намытых хвостов, отложившихся на надводном пляже не далее чем на 100 м от существующего яруса наращивания. Угол откоса дамбы составляет 11 градусов, рис. 1.

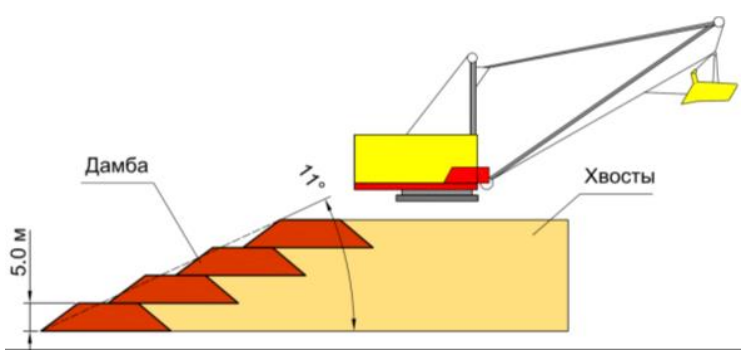

Pис. 1. Схема возведения ограждающей дамбы

Fig. 1. Scheme of the dam construction

\section{Инженерная характеристика объекта}

Окружающий рельеф представляет собой равнину, сильно расчленённую речной и овражно-балочной сетью. В районе гидроузла верхняя часть геологического разреза сложена отложениями четвертичной системы, которые покрывают всю площадь района. В инженерно-геологическом комплексе пород хвостохранилища выделяются три основных типа:
- современные техногенные грунты насыпного и намывного характера (верхняя часть разреза, мощность до 10 м);

- четвертичного и палеогенового возраста различного состава и генезиса (верхняя часть разреза, мощность до 40 м);

- мелового периода - мергельно-меловые карбонатные породы (под четвертичными и намывными породами, местами могут выходить на поверхность, мощность до 70 м).

Насыпные грунты различного состава и мощности имеют место в районах верхних уступов насыпных дамб и плотин. Их мощность от 2,0-3,0 до 6,0-7,0 м. Представлены они песками пылеватыми, кварцевого состава, железистыми, от рыхлых до плотного сложения.

Техногенные грунты намывного характера распространены ниже насыпных грунтов по телу дамб и плотин, а также непосредственно на примыкающих к ним пляжах, служащих основанием наращивания дамб. Грунты отличаются пёстрым литологическим составом, различной степенью консолидации. Они представлены хвостами обогатительной фабрики - в основном пылеватыми, в основном средней плотности и плотными, реже рыхлыми.

Из современных геологических процессов, протекающих на хвостохранилище, на надводных участках пляжей и открытых откосах дамб, характерны периодические размывы, локальные просадки грунтов, а также эоловая эрозия и связанное с ней пылеобразование. Наиболее опасные в этом отношении плотины, находящиеся в стадии наращивания. В настоящее время различным типам атмосферного влияния (воздействие потоков воздуха, осадки, температурные колебания и др.) на геологические процессы и инженерные сооружения уделяется достаточное внимание $[8,19,20]$.

Мел-мергельные породы, расположенные под хвостохранилищем, относятся к полускальным грунтам. При увлажнении и разрушении они способны переходить в пластичное состояние, сопровождаемое резким понижением прочности и приобретением физико-механических свойств связных грунтов. Лабораторные исследования показывают, что в естественном (невыветрелом) состоянии при полном водонасыщении и ступенчатой нагрузке с шагом до 0,6 МПа образцы деформируются незначительно и упруго, а при снятии нагрузки образец полностью восстанавливает объем, как минимум при трех циклах нагружения [21]. Компрессионный модуль деформации мела меняется от 12,5 МПа при давлении 0,1 МПА до 74,0 МПа в интервале давлений от 0,3 до 0,6 МПа [22]. Угол внутреннего трения составляет 33 градуса, удельное сцепление - 202 кПа [23].

Таким образом, характеристики грунтов демонстрируют потенциальную возможность как проявлений потери устойчивости (локальных или масштабных) с одной стороны так и возможность оседания земной поверхности в результате сжатия мелмергельного массива. 


\section{Методика исследования}

\section{Исходные данные}

Исходными данными для анализа являются данные мониторинга деформаций. Для оценки воздействия заполнения хвостохранилища на его внешний контур и окружающую среду был выбран протяженный отрезок времени, в течение которого хорошо документированы скорость заполнения хвостохранилища и уровень деформаций.
Оседания определялись на основе измеренных смещений наблюдательной сети, состоящей из грунтовых и глубинных деформационных марок, смещения определялись геодезическими методами (табл. 1, рис. 2), опорные пункты находятся на расстоянии трехсот метров от нижнего бьефа сооружения.

Нивелирование 3 класса проводится 1 раз в год для контроля оседаний ограждающей плотины, ГНСС измерения в режиме статика проводятся 2 раза в год.

Таблица 1. Состав деформационного мониторинга

Table 1. Deformation monitoring complex

\begin{tabular}{|c|c|c|c|}
\hline $\begin{array}{c}\text { Контролируемый процесс } \\
\text { Controlled process }\end{array}$ & $\begin{array}{l}\text { Количество деформацион- } \\
\text { ных марок } \\
\text { Quantity of deformation marks }\end{array}$ & $\begin{array}{c}\text { Тип деформационных } \\
\text { марок } \\
\text { Type of deformation marks }\end{array}$ & $\begin{array}{c}\text { Метод определения смещений } \\
\text { Method of displacement } \\
\text { measurement }\end{array}$ \\
\hline $\begin{array}{l}\text { Вертикальные смещения } \\
\text { ограждающей дамбы } \\
\text { Subsidence of dyke }\end{array}$ & 197 & $\begin{array}{l}\text { Грунтовые } \\
\text { Ground }\end{array}$ & $\begin{array}{l}\text { Нивелирование } \\
\text { Leveling }\end{array}$ \\
\hline $\begin{array}{l}\text { Вертикальные смещения } \\
\text { головной дамбы } \\
\text { Subsidence of upstream dam bottom }\end{array}$ & 10 & $\begin{array}{c}\text { Грунтовые поверхностные } \\
\text { Ground surface }\end{array}$ & $\begin{array}{l}\text { Нивелирование } \\
\text { Leveling }\end{array}$ \\
\hline $\begin{array}{l}\text { Вертикальные смещения } \\
\text { основания головной дамбы } \\
\text { Subsidence bottom } \\
\text { of upstream dam }\end{array}$ & 9 & $\begin{array}{c}\text { Глубинные } \\
\text { Deep }\end{array}$ & $\begin{array}{l}\text { Нивелирование } \\
\text { Leveling }\end{array}$ \\
\hline $\begin{array}{l}\text { Контроль устойчивости } \\
\text { склонов ограждающей дамбы } \\
\text { Dyke and dam slope stability }\end{array}$ & 63 & $\begin{array}{c}\text { Поверхностные } \\
\text { организованные в створы } \\
\text { lined up }\end{array}$ & $\begin{array}{c}\text { ГНСС измерения, статика } \\
\text { GNSS measurements }\end{array}$ \\
\hline
\end{tabular}

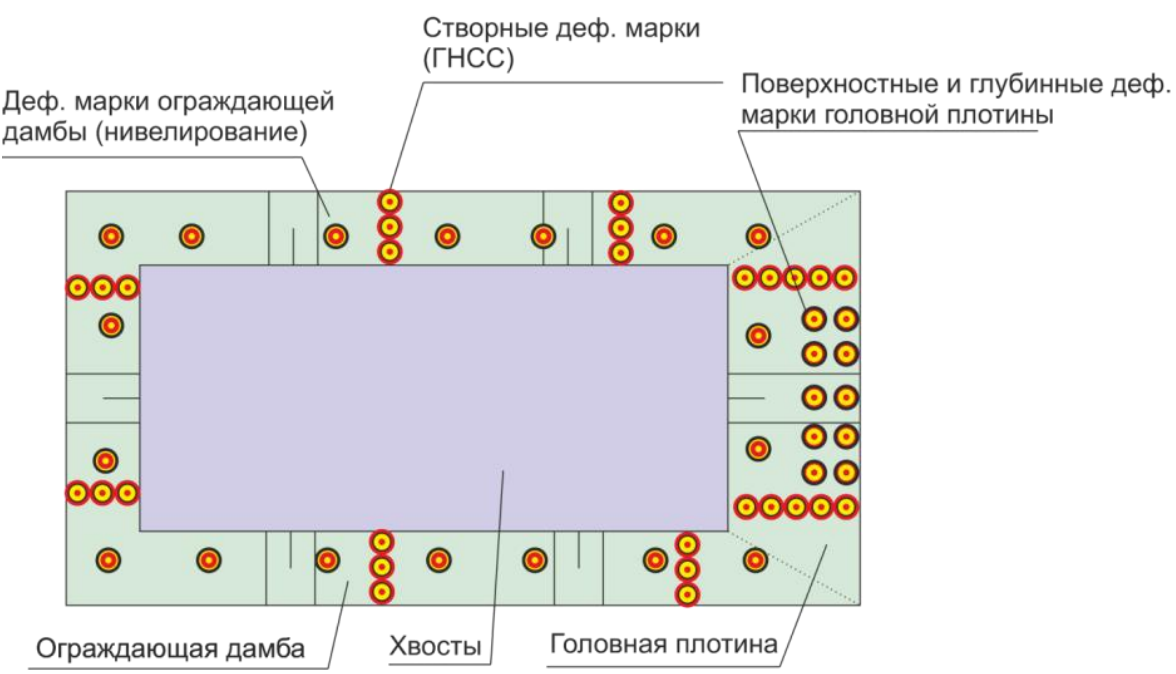

Pис. 2. Схема деформачионного мониторинга

Fig. 2. Scheme of the dam deformation network

В течение выбранного периода времени произведено 8 серий измерений. Результаты каждой серии измерений сравнивались с первой серией выбранного периода. Определялись абсолютные смещения, а также скорости смещений.

Решение поставленных задач

В соответствии с задачами исследований анализировались вертикальные и горизонтальные смещения реперов. В соответствии с природой систематических ошибок отдельно анализировались наблюдения, производимые с помощью геометрического нивелирования и ГНСС измерения. Для грунтовых марок ограждающей дамбы исключались грубые ошибки, определялись приращения вертикальных смещений между сериями измерений и накопленные за все серии.

Для створных наблюдений (осуществляемых с помощью ГНСС) определялись вектора горизонтальных смещений, анализировалось направление и абсолютная величина вектора смещения между сериями и между первой серией и последней.

Для головной плотины получены и проанализированы распределения оседаний глубинных и поверхностных марок, схема заложения приведена на рис. 3 . Оседания глубинных марок при этом интерпретировались как оседания естественного массива в основании сооружения. 


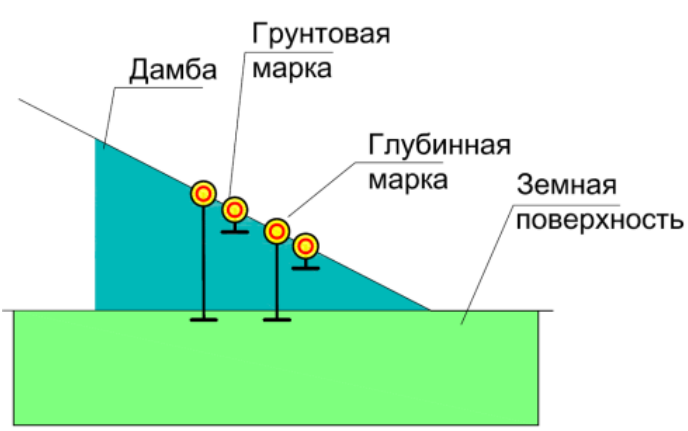

Рис. 3. Схема размещения грунтовых и глубинных марок головной плотины

Fig. 3. Layout of surface and deep deformation marks placed in downstream face

Для получения распределения мощности плотины анализировались две поверхности. Первая - поверхность исходного ландшафта (оврага, в котором началось размещение хвостов), получена путем оцифровки архивных материалов. Вторая поверхность получена на основании измерений уровня воды в прудкахнакопителях и маркшейдерских измерений.

Данные площадного распределения мощности плотины, совместно с фиксируемыми оседаниями деформационных марок ограждающей дамбы были подвергнуты регрессионному анализу. В качестве объясняющей переменной использовалась мощность дамбы, в качестве зависимой - оседания ограждающей дамбы.

Для определения наличия взаимосвязи между приростом массы гидросооружения и оседаниями также использовался регрессионный анализ. В качестве зависимой переменной использовались средние значения оседания дамбы относительно первой серии. Объясняющей переменной выступил прирост массы водохранилища.

Прирост массы определялся как произведение плотности хвостов в обводненном состоянии, изменения уровня воды в отсеках и площади отсеков хвостохранилища.

\section{Результаты}

Анализ результатов ГНСС измерений створных деформационных марок склонов дамбы показывает, что долговременных смещений не выявлено. Наблюдаемая величина смещения за несколько лет примерно равна уровню погрешности измерений в данных условиях. Таким образом, значительные или направленые горизонтальные смещения склонов дамбы остутсвуют.

Фиксируемые вертикальные смещения поверхностных деформационных марок ограждающей дамбы, а также глубинных и поверхностных марок говловной дамбы превышают уровень точности измерений. За исследуемые 8 серий измерений средний уровень оседаний составляет в среднем 51 мм, что соответствует оседанию 5 мм при увеличении уровня воды в отсеках на 1 м. Оседания распределены неравномерно, максимальные оседания зафиксированы в области головной плотины (рис. 4). Величина оседа- ний головной плотины от верхнего бьефа дамбы к нижнему снижается. Минимальные оседания зафиксированы поверхностными марками в области нижнего бьефа головной части плотины, составляют около 10 MM.

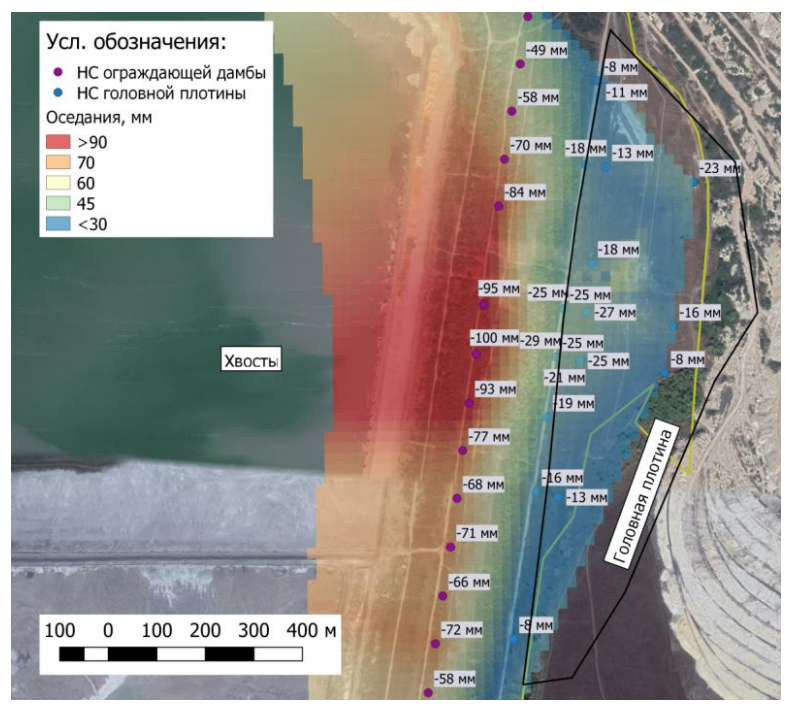

Pис. 4. Распределение оседаний в области головной плотинь

Fig. 4. Subsidence distribution in the head dam area

Вертикальные смещения глубинных и поверхностных марок синхронны и близки по значениям. На первой части рис. 5 показаны оседания глубинных марок, на второй - оседания поверхностных, на третьей - совмещенные. Глубинные и поверхностные марки, в силу утери части марок, покрывают несколько разные участки массива, однако на перекрывающемся участке оседания совпадают. Данные наблюдения показывают, что напорная часть головной плотины деформируется как единое целое с естественным массивом пород.

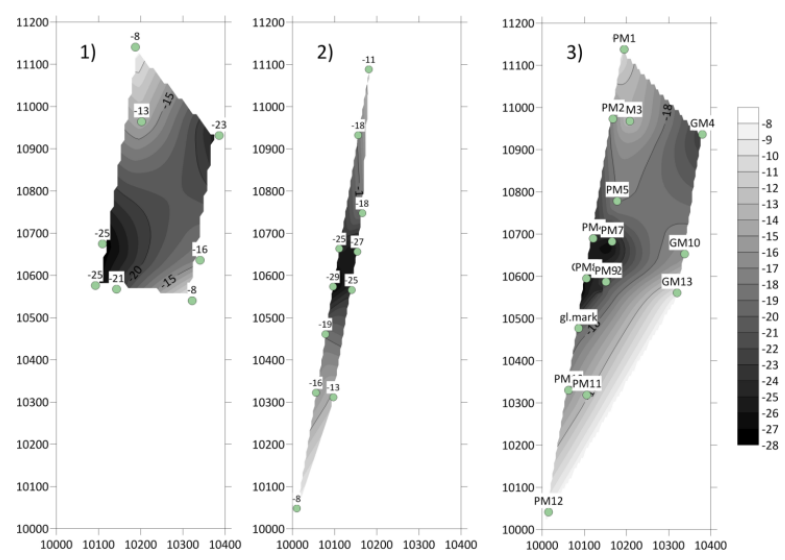

Рис. 5. Рапределение оседаний деформационных марок головной плотины, мм: 1) глубинных марок; 2) поверхностных марок; 3) общее распределение оседаний

Fig. 5. Subsidence distribution of the deformation marks in the head dam area, mm: 1) deep mark; 2) surface marks; 3) complex distribution of subsidence 
Для определения характера смещений ограждающей дамбы (деформации тела дамбы или оседание естественного массива) был проведен анализ распределений мощности дамбы и оседаний деформационных марок ограждающей дамбы. На рис. 6 каждой точке графика соответствует одна деформационная марка ограждающей дамбы. По оси абсцисс отложены значения мощности дамбы в области установки каждой марки, а по оси ординат - оседания марок.

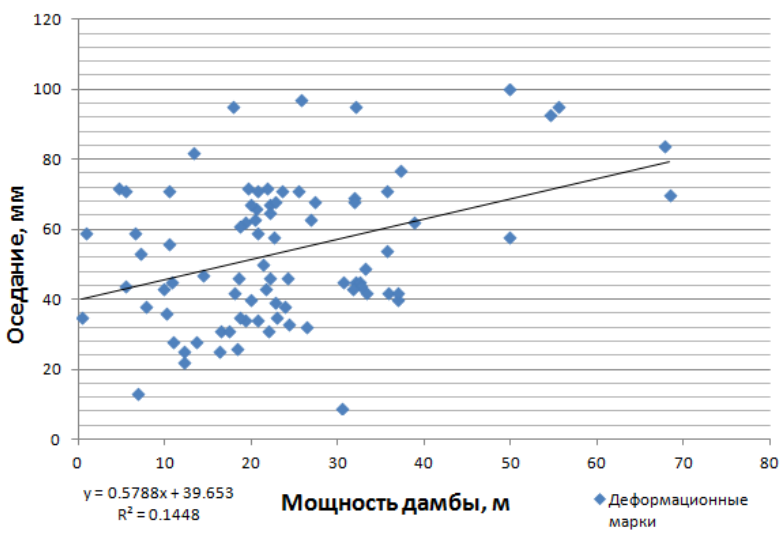

Pис. 6. График линейной регрессии оседаний деформационных марок и мощности дамбы

Fig. 6. Linear regression plot of deformation marks subsidence and dam thickness

Из графика виден существенный разброс в распределениях. Коэффициент детерминации линейной регрессии $\mathrm{R}^{2}$ равен 0,14 . Иными словами, связи между мощностью дамбы и распределением не выявлено. Соответственно, причиной наблюдаемых смещений является сжатие естественного массива, находящегося под сооружением. Однако остается открытым вопрос соответствия максимальных зафиксированных оседаний и набольшей мощности дамбы в районе головной плотны. Возможно наличие тонкой, менее очевидной связи, данный вопрос требует дальнейших исследований.

График зависимости оседаний дамбы от массы складированных хвостов изображен на рис. 7. Каждой точке графика соответствует одна серия измерений. По оси абсцисс отложен прирост массы, который определялся как произведение ежегодного прироста объема хвостохранилища на плотность складированных хвостов в увлажненном состоянии. Значения усредненных оседаний дамбы по сериям измерений отложены по оси ординат, определялись как среднее арифметическое оседаний грунтовых марок ограждающей дамбы хвостохранилища за исключением грубых выбросов измерений. Из графика видно, что ко-

\section{СПИСОК ЛИТЕРАТУРЬ}

1. Foster M., Fell R., Spannagle M. The statistics of embankment dam failures and accidents // Canadian Geotechnical Journal. 2000. - V. 37. - P. 1000-1024. URL: https://doi.org/10.1139/t00030 (дата обращения: 13.03.2020).

2. Design, construction and management of tailings storage facilities for surface disposal in China: case studies of failures / Z. Wei, G. Yin, J.G. Wang, L. Wan, G. Li // Waste Management \& Research. 2013. - V. 31. - P. 106-112. DOI: $10.1177 / 0734242 X 12462281$ эффициент детерминации линейной регрессии $\mathrm{R}^{2}$ составляет 0,86 , что свидетельствует о наличии значимой связи.

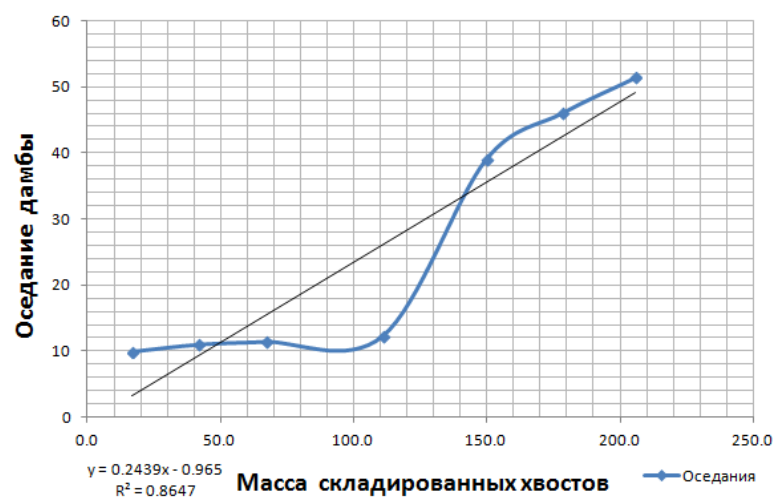

Pис.7. График зависимости оседаний дамбы от массы складированных хвостов, мм

Fig. 7. Graph of the relationship of the dam subsidence and the mass of the stored tailings, $\mathrm{mm}$

\section{Заключение}

Результаты исследования позволили установить характер оседаний ограждающей дамбы и головной плотины хвостохранилища. Установлено, что горизонтальные смещения склонов дамбы хвостохранилища близки к точности определения, а оседания ограждающей дамбы показывают устойчивую связь с приростом массы хвостохранилища. Распределение оседаний марок дамбы хвостохранилища, характер деформирования головной плотины, а также отсутствие значимых горизонтальных смещений позволяют заключить, что тело дамбы находится в устойчивом состоянии, а фиксируемые оседания - проявление деформации естественного массива в основании гидросооружения под нагрузкой веса складируемых хвостов.

Примененный подход в оценке деформирования хвостохранилищ базируется на соотношениях между наблюдаемыми смещениями, характеристиками объекта и интенсивностью складирования отходов. При этом принципиально важно, что нет зависимости между предлагаемой обработкой измерений и методами определения смещений. Это одинаково годится для наземных и спутниковых технологий, лазерного сканирования, InSAR или классического нивелирования. Подход позволяет определять соотношение между опасными постепенным скольжением (с потерей устойчивости верхних слоев дамбы) и безопасными вертикальными смещениями, связанными с деформацией массива горных пород под сооружением.

3. Chronology of major tailings dam failures (from 1960 to 2019). WISE uranium project, tailings dam safety. WISE (World Information Service on Energy). URL: http://www.wiseuranium.org/mdaf.html (дата обращения: 13.03.2020).

4. Characterizing the spatial distribution and fundamental controls of landslides in the three gorges reservoir area, China / S. Li, Q. Xu, M. Tang, J. Iqbal, J. Liu, X. Zhu, F. Liu, D. Zhu // Bulletin of engineering geology and the environment. - 2019. - V. 78. P. 4275-4290. DOI: 10.1007/s10064-018-1404-5 
5. Strength and deformation of tailings with fine-grained interlayers Q. Chen, C. Zhang, C. Yang, C. Ma, Z. Pan, J. Daemen // Engineering geology. - 2019. - V. 256. - P. 110-120. DOI: 10.1016/j.enggeo.2019.04.007

6. Mechanical behavior and particle breakage of tailings under high confining pressure / C. Zhang, Q. Chen, Z. Pan, C. Ma // Engineering geology. - 2020. - V. 265. DOI: 10.1016/j.enggeo.2019.105419

7. Razavi S.K., Bonab M.H., Dabaghian A. Investigation into the internal erosion and local settlement of Esfarayen Earth-Fill Dam // Journal of geotechnical and geoenvironmental engineering. 2020.- V. 146. URL: https://doi.org/10.1061/(ASCE)GT.19435606.0002216 (дата обращения: 13.03.2020).

8. Ageing deformation of tailings dams in seasonally frozen soil areas under freeze-thaw cycles / J. Jin, S. Li, C. Song, X. Zhang, X. Lv // Scientific reports. - 2019. - V. 9. DOI: 10.1038/s41598019-51449-6

9. Meng X. Research on the cause analysis and application of monitoring technology of damping body deformation in tailings reservoir // Proc. of the 2018 International Workshop on Advances in Social Sciences (IWASS 2018). - Fuzhou, China, 2018. P. 545-548. DOI: 10.25236/iwass.2018.112

10. Empirical and semi-analytical models for predicting peak outflows caused by embankment dam failures / B. Wang, Y. Chen, C. Wu, Y. Peng, J. Song, W. Liu, X. Liu // Journal of Hydrology. 2018. - V. 562. - P. 692-702. DOI: 10.1016/j.jhydrol.2018.05.049

11. Mohamed M.M.A. Overtopping breach peak outflow approximation of embankment dam by using Monte Carlo method // Beni-Suef University Journal of Basic and Applied Sciences. - 2018. - V. 7. P. 724-732. URL: https://doi.org/10.1016/j.bjbas.2018.10.002 (дата обращения: 13.03.2020).

12. Froehlich D.C. Peak outflow from breached embankment dam // Journal of Water Resources Planning and Management. - 1995. V. 121. URL: https://doi.org/10.1061/(ASCE)07339496(1995)121:1(90) (дата обрашения: 13.03.2020).

13. Geodetic investigation of landslides and land subsidence: case study of the Bhurkunda coal mines and the Sirobagarh landslide / A. Tiwari, A. Narayan, R. Dwivedi, A. Swadeshi, S. Pasari, O. Dikshit // Surveyreview. - V. 52. - Iss. 371. - P. 134-149. DOI 10.1080/00396265.2018.1531654.

14. Displacements study of an earth fill dam based on high precision geodetic monitoring and numerical modeling / E. Acosta, C. de Lacy, I. Ramos, J. Cano, M. Herrera, M. Aviles, J. Antonio // Sensors. - V. 18. - P. 1369. DOI: 10.3390/s18051369.

15. Estimating and comparing dam deformation using classical and GNSS techniques / R. Barzaghi, N. Cazzaniga, C. Gaetani, L. Pinto, V. Tornatore // Sensors. - V. 18 . - P. 756. DOI: $10.3390 / \mathrm{s} 1803075$
16. Perspectives on the prediction of catastrophic slope failures from satellite InSAR / T. Carla, E. Intrieri, F. Raspini, F. Bardi, P. Farina, A. Ferretti, D. Colombo, F. Novali, N. Casagli // Scientific reports. - 2019. - V. 9. DOI: 10.1038/s41598-01950792-y.

17. Consolidation settlement of Salt Lake County tailings impoundment revealed by time-series InSAR observations from multiple radar satellites / X. Hu, T. Oommen, Z. Lu, T. Wang, J. Kim // Remote sensing of environment. - 2017. - V. 202. P. 199-209. DOI: 10.1016/j.rse.2017.05.023

18. Risk assessment for tailings dams in Brumadinho of Brazil using InSAR time series approach / Z. Du, L. Gea, A. Hay-Man Ng, Q. Zhu, F.G. Horgand, Q. Zhang // Science of The Total Environment. - 2020. - V. 717. URL: https://doi.org/10.1016/j.scitotenv.2020.137125 (дата обращения: 13.03.2020)

19. Wan C.F., Fell R. Investigation of rate of erosion of soils in embankment dams // Journal of Geotechnical and Geoenvironmental Engineering. - 2004. - V. 130. URL: https://doi.org/10.1061/(ASCE)1090-0241(2004)130:4(373) (дата обращения: 13.03.2020).

20. Селезнев А.А., Тетерин А.Ф., Ярмошенко И.В. Метеорологические условия формирования поверхностного стока наносов в период весеннего снеготаяния в городской среде // Известия Томского политехнического университета. Инжиниринг георесурсов. - 2020. - Т. 331. - № 2. - C. 7-16. DOI: $10.18799 / 24131830 / 2020 / 2 / 2476$

21. Колтунов Н.В. Обобщенная характеристика физикомеханических свойств мело-мергельных пород правобережья дона // Студенческий научный форум: V Международная студенческая научная конференция. - Белгород: Белгородский государственный национальный исследовательский университет, 2013. URL: http://scienceforum.ru/2013/article/2013003242 (дата обращения: 13.03.2020).

22. Овчинников А.В., Кучеренко А.П., Аладьин В.П. Прочностные характеристики мелового грунта определенные по результатам испытаний методом одноплоскостного среза // II Всероссийская заочная (с международным участием) научно-практическая конференция молодых ученых, аспирантов и студентов. - Белгород, 2012. - C. 13-16. URL: http://www.lib.tpu.ru/fulltext/c/2013/C11/V1/214.pdf (дата обращения: 13.03.2020).

23. Овчинников А.В. Лабораторные испытания полускального мелового грунта для определения модуля деформации // Новые дороги России. - Саратов: ООО «Издательский центр «Наука», 2011. - С. 233-239.

\section{Информация об авторах}

E中peмов E.Ю., научный сотрудник Института горного дела УрО РАН. 
UDC 622.834

\title{
ANALYSIS OF THE EMBANKMENT DAM DEFORMATION DURING TAILING STORAGE RESERVOIR FILLING
}

\author{
Evgeniy Yu. Efremov, \\ Efremov-eu@mail.ru \\ Institute of Mining of the Ural Branch of the Russian Academy of Sciences, \\ 58, Mamin-Sibiryak street, Yekaterinburg, 620219, Russia.
}

\begin{abstract}
Relevance. Monitoring of earth-filled tailings dams deformation is an important element of industrial safety. The result of deformation monitoring is the defined displacement of dam surface or individual marks of the deformation network. However, not all displacements are dangerous. In this paper, the author justified the separation of displacements into slope deformations, which is a risk of dam failure, and subsidence due to compression of the underlying rock mass that are not dangerous.

The purpose of the research is to assess the mechanical effect of the storing tailings on the surrounding earth-filled dam and the underlying rock mass; determine the prevailing nature of the observed deformation marks displacements - the dam ground layer sliding or subsidence of the earth's surface under the influence of the mass of stored mining waste.

Research methods are geostatistical analysis of vertical and horizontal displacements of the deformation network marks. The displacements of the deformation marks were determined using GNSS measurements and leveling.

Conclusions. It is established that the predominant type of displacements is subsidence. Horizontal displacements are equal to the level of measurement error. Investigation have shown that the observed displacements are not a result of the sliding of the earth-filled dam's material, but subsidence caused by compression of the rock mass under the influence of the weight of the tailings.
\end{abstract}

\section{Key words:}

Earth-filled tailings dams, tailing storage, dam failure, slope stability, deformation network.

\section{REFERENCES}

1. Foster M., Fell R., Spannagle M. The statistics of embankmen dam failures and accidents. Canadian Geotechnical Journal, 2000, vol. 37, pp. 1000-1024. Available at: https://doi.org/10.1139/t00030 (accessed 13 March 2020)

2. Wei Z., Yin G., Wang J.G., Wan L., Li G. Design, construction and management of tailings storage facilities for surface disposal in China: case studies of failures. Waste Management \& Research, 2013, vol. 31, pp. 106-112. DOI: 10.1177/0734242X12462281

3. Chronology of major tailings dam failures (from 1960 to 2019). WISE uranium project, tailings dam safety. WISE (World Information Service on Energy). Available at: http://www.wiseuranium.org/mdaf.html (accessed 13 March 2020).

4. Li S., Xu Q., Tang M., Iqbal J., Liu J., Zhu X., Liu F., Zhu D. Characterizing the spatial distribution and fundamental controls of landslides in the three gorges reservoir area, China. Bulletin of engineering geology and the environment, 2019, vol. 78, pp. 4275-4290. DOI: 10.1007/s10064-018-1404-5

5. Chen Q., Zhang C., Yang C., Ma C., Pan Z., Daemen J. Strength and deformation of tailings with fine-grained interlayers. Engineering geology, 2019, vol. 256, pp. 110-120. DOI: 10.1016/j.enggeo.2019.04.007

6. Zhang C., Chen Q., Pan Z., Ma C. Mechanical behavior and particle breakage of tailings under high confining pressure. Engineering geology, 2020, vol. 265. DOI: 10.1016/j.enggeo.2019.105419

7. Razavi S.K., Bonab M.H., Dabaghian A. Investigation into the internal erosion and local settlement of Esfarayen Earth-Fill Dam. Journal of geotechnical and geoenvironmental engineering, 2020, vol. 146. Available at: https://doi.org/10.1061/(ASCE)GT.19435606.0002216 (accessed 13 March 2020).

8. Jin J., Li S., Song C., Zhang X., Lv X. Ageing deformation of tailings dams in seasonally frozen soil areas under freeze-thaw cycles. Scientific reports, 2019, vol. 9. DOI: 10.1038/s41598-019-51449-6.

9. Meng X. Research on the cause analysis and application of monitoring technology of damping body deformation in tailings reservoir. Proc. of the 2018 International Workshop on Advances in Social Sciences (IWASS 2018). Fuzhou, China, 2018. pp. 545-548. DOI: 10.25236/iwass.2018.112

10. Wang B., Chen Y., Wu C., Peng Y., Song J., Liu W., Liu X. Empirical and semi-analytical models for predicting peak outflows caused by embankment dam failures. Journal of Hydrology, 2018, vol. 562, pp. 692-702. DOI: 10.1016/j.jhydrol.2018.05.049
11. Mohamed M.M.A. Overtopping breach peak outflow approximation of embankment dam by using Monte Carlo method. Beni-Suef University Journal of Basic and Applied Sciences, 2018, vol. 7, pp. 724-732. Available at: https://doi.org/10.1016/j.bjbas. 2018.10.002 (accessed 13 March 2020).

12. Froehlich D.C. Peak outflow from breached embankment dam. Journal of Water Resources Planning and Management, 1995, vol. 121. Available at: https://doi.org/10.1061/(ASCE)07339496(1995)121:1(90) (accessed 13 March 2020).

13. Tiwari A., Narayan A., Dwivedi R., Swadeshi A., Pasari S., Dikshit O. Geodetic investigation of landslides and land subsidence: case study of the Bhurkunda coal mines and the Sirobagarh landslide. Survey review, vol. 52, Iss. 371, pp. 134-149. DOI: 10.1080/00396265.2018.1531654.

14. Acosta E., De Lacy C., Ramos I., Cano J., Herrera M., Aviles M., Antonio J. Displacements study of an earth fill dam based on high precision geodetic monitoring and numerical modeling. Sensors, vol. 18, pp. 1369. DOI: 10.3390/s18051369.

15. Barzaghi R., Cazzaniga N., Gaetani C., Pinto L., Tornatore V. Estimating and comparing dam deformation using classical and GNSS techniques. Sensors, vol. 18, pp. 756. DOI: $10.3390 / \mathrm{s} 1803075$

16. Carla T., Intrieri E., Raspini F., Bardi F., Farina P.P., Ferretti A., Colombo D., Novali F., Casagli N. Perspectives on the prediction of catastrophic slope failures from satellite InSAR. Scientific reports, 2019, vol. 9. DOI: 10.1038/s41598-019-50792-y.

17. Hu X., Oommen T., Lu Z., Wang T., Kim J. Consolidation settlement of Salt Lake County tailings impoundment revealed by timeseries InSAR observations from multiple radar satellites. Remote sensing of environment, 2017, vol. 202, pp. 199-209. DOI: 10.1016/j.rse.2017.05.023

18. Du Z., Gea L., Hay-Man A., Ng, Zhu Q., Horgand F.G., Zhang Q. Risk assessment for tailings dams in Brumadinho of Brazil using InSAR time series approach. Science of The Total Environment, 2020, vol. 717. Available at: https://doi.org/10.1016/ j.scitotenv.2020.137125 (accessed 13 March 2020).

19. Wan C.F., Fell R. Investigation of rate of erosion of soils in embankment dams. Journal of Geotechnical and Geoenvironmental Engineering, 2004, vol. 130. Available at: https://doi.org/10.1061/(ASCE)1090-0241(2004)130:4(373) (accessed 13 March 2020).

20. Selesnev A.A., Teterin A.F., Yarmosenko I.V. Meteorological conditions of surface sediment runoff formation during spring 
snowmelt in urban environment. Bulletin of the Tomsk Polytechnic University. Geo Assets Engineering, 2020, vol. 331, no. 2, pp. 7-16. In Rus. DOI: 10.18799/24131830/2020/2/2476.

21. Koltunov N.V. Obobshchennaya kharakteristika fizikomekhanicheskikh svoystv melo-mergelnykh porod pravoberezhya dona [A generalized characteristic of the physicomechanical properties of the fine marl rocks of the right bank of the Don]. $V$ Mezhdunarodnaya studencheskaya nauchnaya konferentsiya. Studencheskiy nauchny forum [V International Student Scientific Conference. Student Scientific Forum]. Belgorod, Belgorod national university, 2021. Available at: http://scienceforum.ru/ 2013/article/2013003242 (accessed 13 March 2020).

22. Ovchinnikov A.V., Kucherenko A.P., Aladin V.P. Prochnostnye lharakteristiki melovogo grunta opredelennye po rezultatam ispytaniy metodom odnoploskostnogo sreza [Resisting power of cretaceous soils using shear test]. II Vserossiyskaya zaochnaya (s mezhdunarodnym uchastiem) nauchno-prakticheskaya konferentsiya molodykh uchenykh, aspirantov i studentov [II All-Russian correspondence (with international participation) scientific and practical conference of young scientists, graduate students and students]. Belgorod, 2012. pp. 13-16. Available at: http://www.lib.tpu.ru/fulltext/c/2013/C11/V1/214.pdf (accessed 13 March 2020).

23. Ovchinnikov A.V. Laboratornye ispytaniya poluskalnogo melovogo grunta dlya opredeleniya modulya deformatsii [Laboratory tests of semi-rocky chalky soil for determining the deformation modulus]. Novye dorogi Rossii [New Roads of Russia]. Saratov, Nauka” Publ. LLC, 2011. pp. 233-239.

Received: 19 April 2021.

\section{Information about the authors}

Evgeniy Yu. Efremov, researcher, Institute of Mining of the Ural Branch of the Russian Academy of Sciences. 